Chinese Journal of Organic Chemistry

\title{
一种咪唑并吩嗪内酰胺反应型识别氰离子的苂光探针
}

\author{
李翔林奇曲文娟李乔程晓斌李文婷 \\ 张有明姚虹魏太保* \\ (西北师范大学化学化工学院 教育部生态环境相关材料重点实验室 甘肃省高分子材料重点实验室 兰州 730070)
}

\begin{abstract}
摘要 设计合成了一种新型咪坐并吩嗪内酰胺苂光传感器分子 $(\mathbf{S 1})$, 通过核磁共振氢谱、碳谱和高分辨质谱等手段对其 结构进行了表征, 并测定了 $\mathbf{S 1}$ 在二甲基亚砜(DMSO)溶液中的荧光光谱, 其最大苂光发射波长为 $524 \mathrm{~nm}$. S1 的 DMSO 溶液具有亮黄色荧光. 当在 $\mathbf{S 1}$ 的 DMSO 溶液中分别加入 $\mathrm{F}^{-}, \mathrm{Cl}^{-}, \mathrm{Br}^{-}, \mathrm{I}^{-}, \mathrm{AcO}^{-}, \mathrm{H}_{2} \mathrm{PO}_{4}^{-}, \mathrm{HSO}_{4}^{-}, \mathrm{ClO}_{4}^{-}$和 $\mathrm{SCN}^{-}$等阴 离子后, 发现只有 $\mathrm{CN}^{-}$的加入使 $\mathbf{S} 1$ 的荧光光谱出现明显的下降并发生红移. 其溶液荧光颜色由黄色变为橘红色, 说明 S1 对 $\mathrm{CN}^{-}$具有良好的专一选择性. 抗干扰实验结果表明, 这一识别过程不受其它阴离子干扰. 通过计算得到, S1 对 CN 的苂光光谱最低检测限为 $9.96 \times 10^{-7} \mathrm{~mol} / \mathrm{L}$, 这一数值低于世界卫生组织所规定的饮用水中氰离子含量. 机理研究表 明, $\mathbf{S} 1$ 是一种反应型识别 $\mathrm{CN}^{-}$的荧光传感器. 此外, 将 $\mathrm{S} 1$ 负载于固态硅胶之上, 制备成固体 $\mathrm{CN}^{-}$识别材料, 并成功用 于对固体 $\mathrm{NaCN}$ 的识别和对 $\mathrm{CN}^{-}$水溶液的检测.
\end{abstract}

关键词 吩嗪衍生物; 苂光传感器; 阴离子识别

\section{A Novel Imidazophenazine Lactam Reaction Type Recognition Cyanide Ion Fluorescence Probe}

\author{
Li, Xiang \\ Lin, Qi \\ Qu, Wenjuan \\ Li, Qiao \\ Chen, Xiaobin \\ Li, Wenting \\ Zhang, Youming \\ Yao, Hong \\ Wei, Taibao* \\ (College of Chemistry and Chemical Engineering, Northwest Normal University, Lanzhou 730070)
}

\begin{abstract}
A novel imidazophenazine lactam fluorescence chemosensor (S1) was designed, synthesized and characterized by ${ }^{1} \mathrm{H}$ NMR, ${ }^{13} \mathrm{C}$ NMR and HRMS techniques. Fluorescence spectra of $\mathbf{S 1}$ in dimethyl sulfoxide (DMSO) solution were measured. Its maximum emission wavelength was $524 \mathrm{~nm}$. The DMSO solution of $\mathbf{S 1}$ has bright yellow fluorescence. Respectively, to the $\mathrm{S} 1$ solution add $\mathrm{F}^{-}, \mathrm{Cl}^{-}, \mathrm{Br}^{-}, \mathrm{I}^{-}, \mathrm{AcO}^{-}, \mathrm{H}_{2} \mathrm{PO}_{4}^{-}, \mathrm{HSO}_{4}^{-}, \mathrm{ClO}_{4}^{-}$and $\mathrm{SCN}^{-}$, the fluorescent color of the solution didn't change. Only the $\mathrm{CN}^{-}$addition, the fluorescent color of the $\mathbf{S 1}$ solution changed from yellow to orange-red, indicating that $\mathbf{S 1}$ has good specific selectivity for $\mathrm{CN}^{-}$. The results of anti-disturbance experiment demonstrated that $\mathbf{S 1}$ detect $\mathrm{CN}^{-}$ without interference from other anions. By calculation, the linear of the fluorescence of the sensor for $\mathrm{CN}^{-}$is $9.96 \times 10^{-7}$. This value is lower than the World Health Organization (WHO) provisions of the cyanide content of drinking water. Mechanism studies show that $\mathbf{S} 1$ is a fluorescence sensor by reactive recognition $\mathrm{CN}^{-}$. In addition, the application of sensor $\mathbf{S} 1$ supported on solid silica gel was used as a solid material to detecting solid $\mathrm{NaCN}$ and $\mathrm{CN}^{-}$in pure water.

Keywords phenazine derivative; fluorescence sensor; detecting anions
\end{abstract}

吩嗪及其衍生物组成了一大类含氮杂环化合物 ${ }^{[1]}$, 它们广泛存在于自然界中, 对这类天然产物的分离鉴别 以及合成研究一直受到学者的关注 ${ }^{[2]}$. 就合成方法而言, 它们可以由各种各样的细菌产生, 也可以由人工合成, 具有合成简单、易于修饰等特点. 吩嗪衍生物具有较大
的共轭系统，苂光量子产率较高、发射谱带较窄、斯托 克斯位移较大、最大吸收与发射波长在可见光区，并且， 易于被质子化具有一定的电子接受能力 ${ }^{[3,4]}$. 因而吩嗪 类化合物被广泛应用于生物、医学及有机光电材料领域 中 $^{[5 \sim 7]}$. 近年来，吩嗪衍生物在超分子化学领域应用也

\footnotetext{
* Corresponding author. E-mail: weitaibao@126.com

Received November 3, 2016; revised December 29, 2016; published online January 17, 2017.

Project supported by the National Natural Science Foundation of China (Nos. 21662031, 21661028, 21574104, 21262032).

国家自然科学基金(Nos. 21662031, 21661028, 21574104, 21262032)资助项目.
} 
受到关注, 研究者们发现吩嗪具有良好的荧光发色基 团，可用于对阳离子、阴离子和中性分子的识别基团 ${ }^{[8,9]}$. 因此研究吩嗪类化合物在有机菼光离子识别传感器方 面的新应用具有重要意义.

荧光传感器是能够将分子识别事件通过分子的荧 光信号有效表达出来的分子器件 ${ }^{[10 ~ 15]}$. 近年来, 氰化物 荧光传感器已经发展成为超分子化学中最为活跃的研 究领域之一 ${ }^{[16 ~ 18]}$. 由于氰化物具有很强的毒性, 因此对 我们的生存环境造成了巨大危害 ${ }^{[19]}$. 例如: 工业纤维制 造, 黄金提取和电镀过程中所排放的含氯废水、废气， 以及在杀虫剂和除草剂中所排放和使用的氰化物, 都对 我们日常饮食、饮水以及大气造成严重污染 ${ }^{[20 ~ 22]}$; 甚至, 汽车尾气和烟草燃烧时产生的烟雾中也都含有氰化 物 ${ }^{[23]}$. 据测量: 人在吸烟时, 血液中的氰化物含量会显 著升高 ${ }^{[24]}$. 此外, 一些食物当中也含有氰化物. 例如: 木薯、发芽土豆、苦杏仁以及一些水果的种子当中 ${ }^{[25,26]}$. 少量的氰根离子可以与人体血红细胞结合进而在短时 间内使人窒息死亡 ${ }^{[27]}$. 根据世界卫生组织(WHO)规定, 日常饮用水中 $\mathrm{CN}^{-}$含量不得高于 $1.9 \mu \mathrm{mol} \cdot \mathrm{L}^{-1[28,29]}$. 因 此, 合成简单、高选择性、高灵敏度的氰化物荧光传感 器显得十分重要, 长期以来受到研究者们的重视.

此外, 为了解决苂光传感器分子在实际应用过程中 存在的一些问题, 拓展其应用范围, 通过物理或化学方 法将荧光传感器分子固载到固体材料上是离子苂光化 学传感器发展成为新型实用型材料的一种有效方法. 这 种方法可以将高选择性、高灵敏度、可视化等优良性能 的苂光传感器分子与载体材料的特性相结合, 从而实现 荧光材料在离子识别、吸附和生物应用的多功能化、高 性能化以及检测过程的可视化 ${ }^{[30]}$. 鉴于此, 在我们多年 来研究超分子化学及分子离子识别的基础上 ${ }^{[31 ~ 41]}$, 通 过 2,3-二氨基吩嗪与 1,8-萗二甲酸酐反应，合成了一种 新型咪唑并吩嗪内酰胺荧光传感器 S1, 研究了其阴离 子识别性能, 结果发现, 其对 $\mathrm{CN}^{-}$具有专一选择性识别 功效, 是一种反应型识别 $\mathrm{CN}^{-}$的荧光传感器分子. 此外, 我们将它负载于固态硅胶之上, 这种负载了苂光传感器 分子 $\mathbf{S 1}$ 的固体材料不仅对固体的 $\mathrm{NaCN}$ 具有比色识别 效果, 而且对纯水相中的氰根离子也有同样的效果, 是 一种检测的新材料.

\section{1 结果与讨论}

\section{1 荧光传感器分子的构筑}

苂光传感器分子 $\mathbf{S 1}$ 是以 2,3 -二氨基吩嗪为母体, 1,8 -萗二酸酐为修饰基团的荧光分子. Scheme 1 为荧光 传感器分子 $\mathbf{S 1}$ 的合成路线.

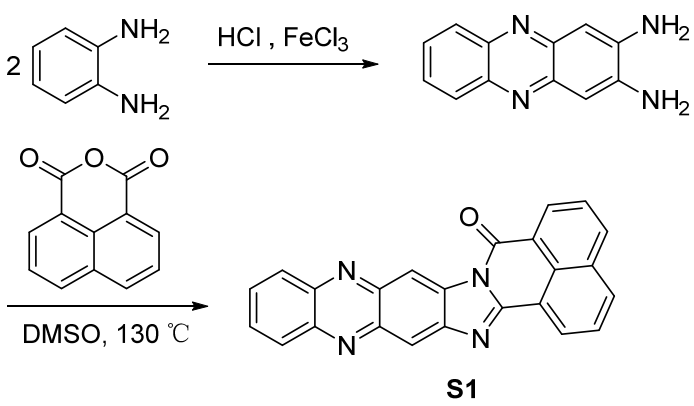

图式 1 菼光传感器分子 $\mathbf{S 1}$ 的合成路线

Scheme 1 Synthetic route of the fluorescence chemosensor S1

\section{2 反应条件探索}

根据文献报道，邻苯二胺可以与环状酸酐或二元酰 氯等酰化试剂反应，得到不同的环状产物，其中有：苯 并咪唑, 环状内酰胺以及苯并咪唑的 $N$-酰化内酰胺类 化合物等产物 ${ }^{[42 ~ 47]}$. 为此我们对邻苯二胺与 1,8 -萗二酸 酐的反应条件进行了考察, 结果见表 1 .

表 1 反应条件的优化

Table1 Optimization of reaction conditions

\begin{tabular}{cccccc}
\hline Entry & Molar ratio $^{a}$ & $t /{ }^{\circ} \mathrm{C}$ & $t / \mathrm{h}$ & Solvent & Yield\% \\
\hline 1 & $1: 1.0$ & 80 & 8 & DMF & 22.2 \\
2 & $1: 1.2$ & 80 & 8 & DMF & 29.0 \\
3 & $1: 1.5$ & 80 & 8 & DMF & 30.5 \\
4 & $1: 1.2$ & 100 & 8 & DMF & 34.1 \\
5 & $1: 1.2$ & 120 & 8 & DMF & 43.3 \\
6 & $1: 1.2$ & 130 & 8 & DMF & 44.3 \\
7 & $1: 1.2$ & 130 & 12 & DMF & 49.5 \\
8 & $1: 1.2$ & 130 & 18 & DMF & 56.7 \\
9 & $1: 1.2$ & 130 & 24 & DMF & 60.2 \\
10 & $1: 1.2$ & 130 & 24 & DMSO & 63.4 \\
\hline${ }^{a} n(2,3$-二氨基吩嗪 $): n(1,8$-萗二酸䣶). & & &
\end{tabular}

首先考察了投料比对产率的影响. 由表 1 可以看出, 随着 1,8-荎二酸酐的物质的量增大，反应产物的产率逐 渐升高(表 1, Entries 1 3). 然而, 当 2,3-二氨基吩嗪与 1,8 -菜二酸䣶的物质的量比为 $1: 1.5$ 时, 相较 $1: 1.2$ 倍 时, 产率只提高了 $1 \%$ 左右. 在产率提高不是很大的情 况下, 考虑到成本控制的因素, 选取 2,3-二氨基吩嗪与 1,8-菜二酸酐的物质的量比为 $1: 1.2$. 升高温度同样有 利于产物的生成(表 1, Entries 2, 4 6), 但考虑到实验室 安全问题，没有继续提升温度，只选择反应温度为 $130{ }^{\circ} \mathrm{C}$. 之后，又对反应时间进行考察，当反应时间为 $24 \mathrm{~h}$ 时, 产率最高(表 1, Entries 6 9). 最后将溶剂由 DMF 换为 DMSO 发现产率有所提高(表 1 , Entries 9, 10). 最终确定合成 S1 的最佳反应条件为: 2,3-二氨基吩 嗪与 2,3 -二氨基吩嗪的物质的量比为 $1: 1.2$, 温度为 $130{ }^{\circ} \mathrm{C}$, 反应时间为 $24 \mathrm{~h}$, 溶剂为 DMSO. 


\section{3 含水量对传感器分子 S1 荧光的影响}

不同的含水量对荧光传感器分子的荧光具有较大 影响. 含水量对传感器分子 $\mathbf{S 1}$ 的苂光强度影响如图 1 和图 2 所示. 从图中可以看到, 当含水量从 $0 \%$ 增加到 $10 \%$ 时, S1 的苂光强度随溶液中含水量的增加而减弱. 此时水的加入使 $\mathbf{S 1}$ 产生堆积, 但这种堆积较为无序, 因 此导致荧光减弱 ${ }^{[48,49]}$. 当含水量从 $10 \%$ 增加到 $30 \%$ 时, S1 的苂光强度随溶液中含水量的增加而增强 ${ }^{[50,51]}$. 此 时的 S1 堆积相对较为有序, 进而产生聚集态荧光, 因此 导致苂光增强. 当含水量从 $30 \%$ 增加到 $90 \%$ 时, S1 的苂 光强度随溶液中含水量的增加而减弱, 直到猝灭. 说明 此时大量的水使得 $\mathbf{S} 1$ 发生了水解，导致荧光猝灭.

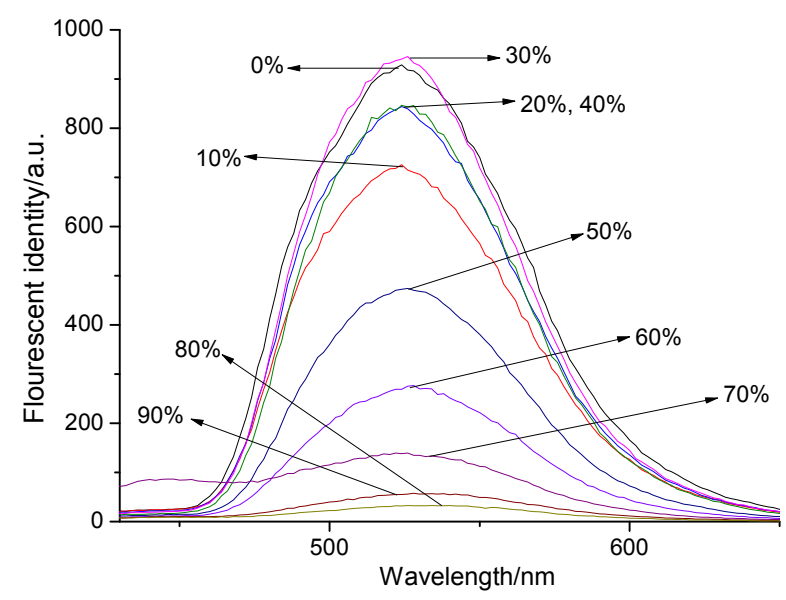

图 1 含水量对传感器分子 $\mathbf{S 1}$ 苂光强度的影响

Figure 1 Fluorescence emission spectrum of chemosensor S1 versus the water content

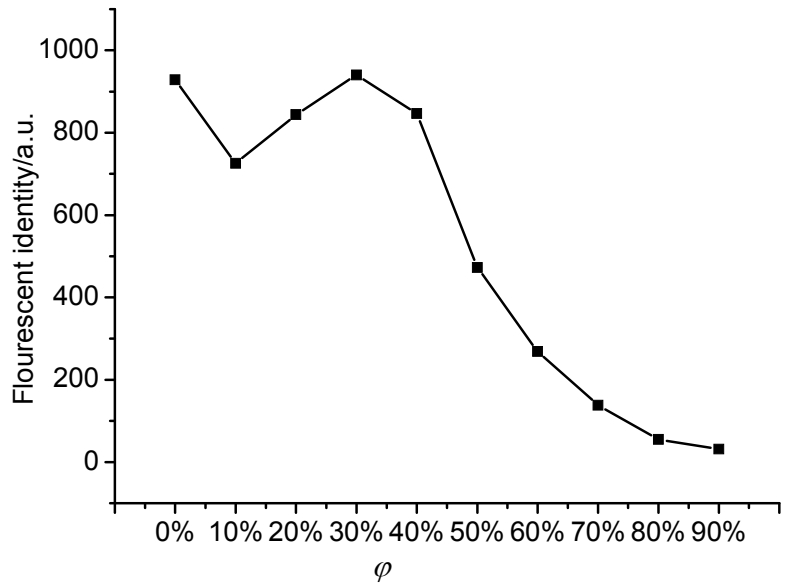

图 2 含水量对传感器分子 $\mathbf{S 1}$ 荧光最大峰变化图

Figure 2 Flourescent identity of chemosensor S1 at $524 \mathrm{~nm}$ versus the water content

\section{4 荧光传感器分子 $\mathrm{S} 1$ 对 $\mathrm{CN}^{-}$的识别}

由以上结果可以看出, 含水量对传感器分子 $\mathrm{S} 1$ 荧 光的影响较大. 为了尽量减免这种影响, 将苂光传感器
分子 $\mathrm{S1}$ 配制成浓度为 $2.0 \times 10^{-5} \mathrm{~mol} / \mathrm{L}$ 的 DMSO 溶液, 并分别向其中加入 50 倍化学计量的各种阴离子 $\left(\mathrm{F}^{-}\right.$, $\mathrm{Cl}^{-}, \mathrm{Br}^{-}, \mathrm{I}^{-}, \mathrm{AcO}^{-}, \mathrm{H}_{2} \mathrm{PO}_{4}^{-}, \mathrm{HSO}_{4}^{-}, \mathrm{ClO}_{4}^{-}, \mathrm{CN}^{-}$和 $\mathrm{SCN}^{-}$). 当加入 $\mathrm{F}^{-}, \mathrm{AcO}^{-}$和 $\mathrm{H}_{2} \mathrm{PO}_{4}^{-}$三种离子时, 对传感 器分子 $\mathrm{S} 1$ 的溶液有轻微的影响, 而 $\mathrm{CN}^{-}$的加入使溶液 颜色变化明显, 如图 3 所示. 说明传感器分子 S1 在自然 光条件下对 $\mathrm{CN}^{-}$不具有十分良好的专一选择性. 然后我 们进行了苂光测定, 其荧光光谱图见图 4. 可以看出, S1 在 $524 \mathrm{~nm}$ 处出现了一个明显的宽苂光发射峰 $\left(\lambda_{\mathrm{ex}}=385\right.$ $\mathrm{nm})$. 当加入 $\mathrm{CN}^{-}$时, $524 \mathrm{~nm}$ 处的苂光发射峰大幅度降 低并发生红移. 此外, 除 $\mathrm{SCN}^{-}$离子的加入使得 $\mathbf{S} 1$ 荧光 光谱略微减弱, 其它阴离子 $\left(\mathrm{F}^{-}, \mathrm{Cl}^{-}, \mathrm{Br}^{-}, \mathrm{I}^{-}, \mathrm{AcO}^{-}\right.$, $\mathrm{H}_{2} \mathrm{PO}_{4}^{-}, \mathrm{HSO}_{4}^{-}$和 $\left.\mathrm{ClO}_{4}^{-}\right)$的加入对 $\mathbf{S 1}$ 溶液的荧光强度 几乎没有改变, 相应的在三用紫外分析仪下可以裸眼观 察到加入 $\mathrm{CN}^{-}$后, 溶液的苂光颜色由亮黄色变为橘红 色，而其它阴离子的对溶液荧光颜色没有影响(图 5). 这 说明传感器分子 $\mathbf{S 1}$ 对 $\mathrm{CN}^{-}$具有很好的专一选择性, 可 以实现在 DMSO 溶液中对 $\mathrm{CN}^{-}$的专一选择性识别.

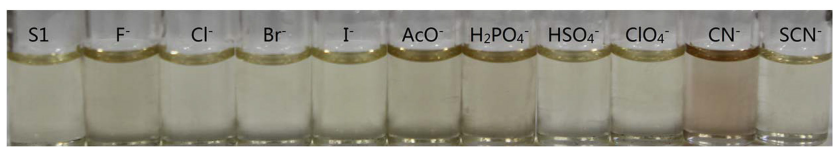

图 3 在传感器分子 $\mathbf{S 1}\left(c=2.0 \times 10^{-5} \mathrm{~mol} / \mathrm{L}\right)$ 的 $\mathrm{DMSO}$ 溶液中 分别加入不同阴离子 $\mathrm{F}^{-}, \mathrm{Cl}^{-}, \mathrm{Br}^{-}, \mathrm{I}^{-}, \mathrm{AcO}^{-}, \mathrm{H}_{2} \mathrm{PO}_{4}^{-}, \mathrm{HSO}_{4}^{-}$, $\mathrm{ClO}_{4}^{-}, \mathrm{CN}^{-}$和 $\mathrm{SCN}^{-}\left(c=1.0 \times 10^{-2} \mathrm{~mol} / \mathrm{L}\right)$ 的照片

Figure 3 Photograph of chemosensor $\mathbf{S 1}\left(c=2.0 \times 10^{-5} \mathrm{~mol} / \mathrm{L}\right)$ in DMSO solution were added to 50 times the stoichiometric amount of various anions $\mathrm{F}^{-}, \mathrm{Cl}^{-}, \mathrm{Br}^{-}, \mathrm{I}^{-}, \mathrm{AcO}^{-}$, $\mathrm{H}_{2} \mathrm{PO}_{4}^{-}, \mathrm{HSO}_{4}^{-}, \mathrm{ClO}_{4}^{-}, \mathrm{CN}^{-}$and $\mathrm{SCN}^{-}\left(c=1.0 \times 10^{-2} \mathrm{~mol} / \mathrm{L}\right)$

\section{5 荧光传感器分子 S1 对选择性识别 $\mathrm{CN}^{-}$的抗干扰 实验}

抗干扰性能对苂光离子传感器选择性识别来说，是 一个非常重要的指标. 为了研究传感器分子 $\mathbf{S} 1$ 对 $\mathrm{CN}^{-}$ 选择性识别的抗干扰能力, 我们做了如下抗干扰实验: 将 50 倍化学计量的 $\mathrm{F}^{-}, \mathrm{Cl}^{-}, \mathrm{Br}^{-}, \mathrm{I}^{-}, \mathrm{AcO}^{-}, \mathrm{H}_{2} \mathrm{PO}_{4}^{-}$, $\mathrm{HSO}_{4}^{-}, \mathrm{ClO}_{4}^{-}$和 $\mathrm{SCN}^{-}$分别加入到传感器分子 $\mathrm{S} 1$ 的溶 液中, 进行荧光光谱测定, 结果发现, 除了 $\mathrm{SCN}^{-}$的加 入使得 S1 溶液苂光略微降低, 其余阴离子的加入对 S1 溶液荧光强度几乎没有影响. 再将等量的 $\mathrm{CN}^{-}$分别加入 到上述体系中, 此时溶液在 $524 \mathrm{~nm}$ 处的荧光强度降低. 取最大荧光发射峰处 $(524 \mathrm{~nm})$ 的数据作柱状抗干扰图 (图 6, $\lambda_{\mathrm{ex}}=385 \mathrm{~nm}$ ). 从图 6 可以看出, 传感器分子 $\mathbf{S 1}$ 对 $\mathrm{CN}^{-}$的识别在其它阴离子加入后没有受到影响, 说明传 感器分子 $\mathbf{S 1}$ 对 $\mathrm{CN}^{-}$的识别具有良好的抗干扰能力.

\section{6 荧光传感器分子 $\mathrm{S} 1$ 识别 $\mathrm{CN}^{-}$的机理探讨}

为了初步探讨传感器分子对 $\mathrm{CN}^{-}$的识别机理, 我们 


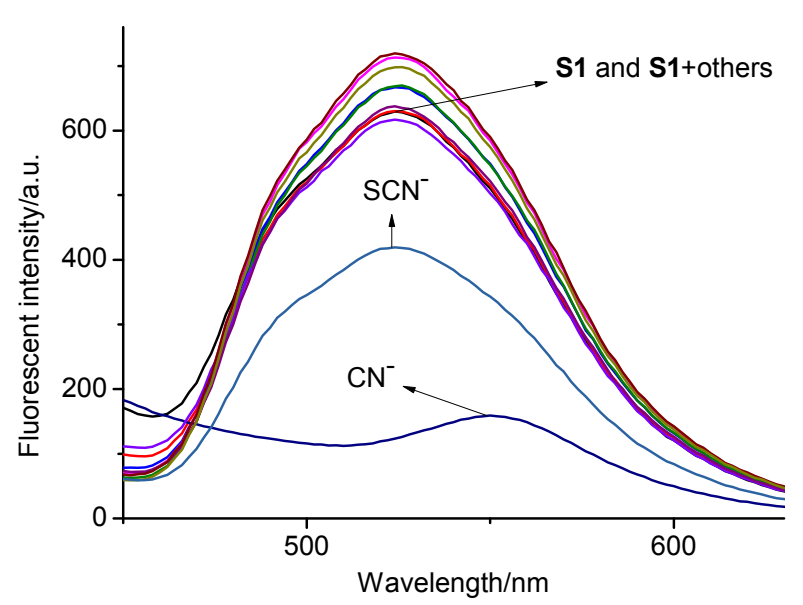

图 4 在传感器分子 $\mathbf{S 1}\left(c=2.0 \times 10^{-5} \mathrm{~mol} / \mathrm{L}\right)$ 的 $\mathrm{DMSO}$ 溶液中 分别加入 50 倍化学计量的各种阴离子 $\mathrm{F}^{-}, \mathrm{Cl}^{-}, \mathrm{Br}^{-}, \mathrm{I}^{-}, \mathrm{AcO}^{-}$, $\mathrm{H}_{2} \mathrm{PO}_{4}^{-}, \mathrm{HSO}_{4}^{-}, \mathrm{ClO}_{4}^{-}, \mathrm{CN}^{-}$和 $\mathrm{SCN}^{-}\left(c=1.0 \times 10^{-2} \mathrm{~mol} / \mathrm{L}\right)$ 的苂光发射光谱

Figure 4 Fluorescence emission spectrum of chemosensor S1 $\left(c=2.0 \times 10^{-5} \mathrm{~mol} / \mathrm{L}\right)$ in DMSO solution were added to 50 times the stoichiometric amount of various anions $\mathrm{F}^{-}, \mathrm{Cl}^{-}, \mathrm{Br}^{-}, \mathrm{I}^{-}$, $\mathrm{AcO}^{-}, \mathrm{H}_{2} \mathrm{PO}_{4}^{-}, \mathrm{HSO}_{4}^{-}, \mathrm{ClO}_{4}^{-}, \mathrm{CN}^{-}$and $\mathrm{SCN}^{-}(c=1.0 \times$ $10^{-2} \mathrm{~mol} / \mathrm{L}$ )

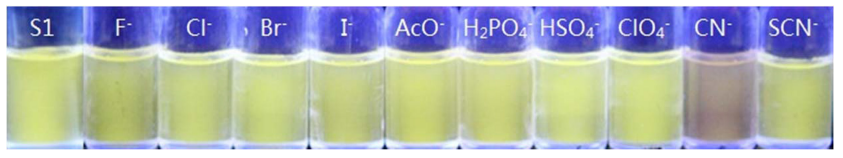

图 5 在传感器分子 $\mathbf{S 1}$ 的 $\left(c=2.0 \times 10^{-5} \mathrm{~mol} / \mathrm{L}\right) \mathrm{DMSO}$ 溶液中 分别加入不同阴离子 $\mathrm{F}^{-}, \mathrm{Cl}^{-}, \mathrm{Br}^{-}, \mathrm{I}^{-}, \mathrm{AcO}^{-}, \mathrm{H}_{2} \mathrm{PO}_{4}^{-}, \mathrm{HSO}_{4}^{-}$, $\mathrm{ClO}_{4}^{-}, \mathrm{CN}^{-}$和 $\mathrm{SCN}^{-}\left(c=1.0 \times 10^{-2} \mathrm{~mol} / \mathrm{L}\right)$ 的比色效果图

Figure 5 Colorimetric renderings of chemosensor S1 $(c=2.0 \times$ $10^{-5} \mathrm{~mol} / \mathrm{L}$ ) in DMSO solution were added to 50 times the stoichiometric amount of various anions $\mathrm{F}^{-}, \mathrm{Cl}^{-}, \mathrm{Br}^{-}, \mathrm{I}^{-}, \mathrm{AcO}^{-}$, $\mathrm{H}_{2} \mathrm{PO}_{4}^{-}, \mathrm{HSO}_{4}^{-}, \mathrm{ClO}_{4}^{-}, \mathrm{CN}^{-}$and $\mathrm{SCN}^{-}\left(c=1.0 \times 10^{-2}\right.$ $\mathrm{mol} / \mathrm{L}$ )

对苂光传感器分子 $\mathbf{S 1}$ 和 $\mathbf{S 1}+\mathrm{CN}^{-}$做了苂光光谱对比, 发现当 $\mathrm{CN}^{-}$加入后, 传感器分子 $\mathbf{S 1}$ 在 $524 \mathrm{~nm}$ 处的最大 发射峰明显降低并且发生红移. 通过质谱检测发现: 在 $\mathrm{S1}+\mathrm{NaCN}$ 体系中, 从质谱图上可以得到在 413.2237 处 出现了 $[\mathrm{M}+\mathrm{H}]^{+}$分子离子峰, 通过分析我们认为是由苂 光传感器分子 $\mathbf{S 1}$ 与 $\mathrm{CN}^{-}$进行亲核加成反应生成相应的 酰基氰, 后者不稳定, 进一步水解, 生成相应的羧酸, 进而转为相应的钠盐, 该钠盐的分子量为 412.3838 .

为了进一步弄清楚传感器分子对 $\mathrm{CN}^{-}$的识别机理, 根据量子化学计算对传感器分子 $\mathrm{S} 1$ 做了分子表面静电 势分析(图 7). 结果显示: 苂光传感器分子 $\mathbf{S 1}$ 表面静电 势负值的极大点在羰基 $\mathrm{C}$ 原子附近, 其值为 $-1.84 \mathrm{eV}$. 说明苂光传感器分子 $\mathbf{S 1}$ 的羰基碳原子不稳定、反应活 性高，容易发生亲核加成反应导致开环.

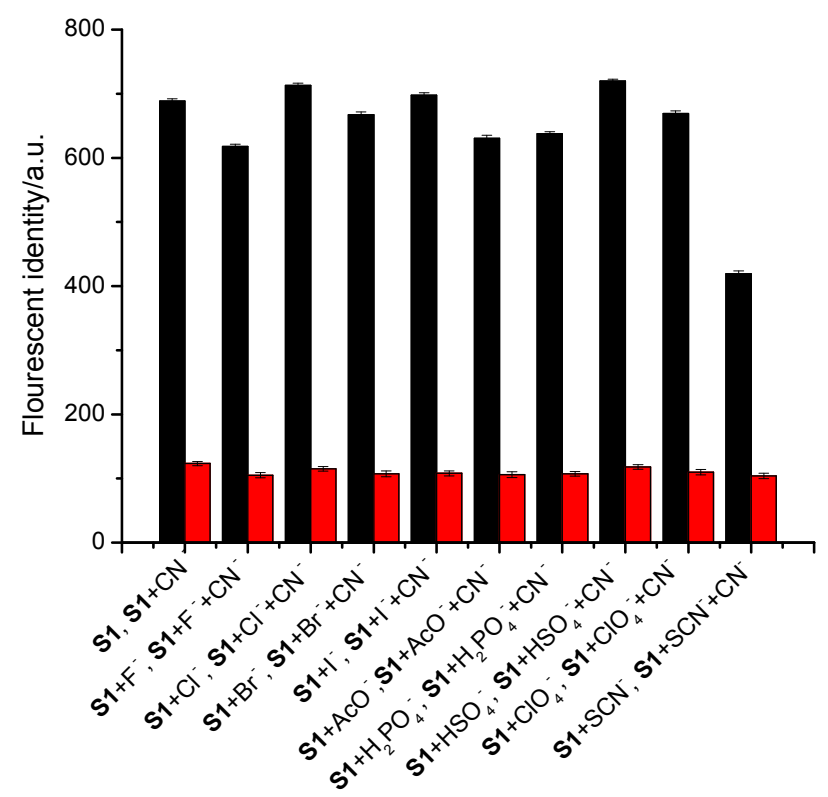

图 6 苂光传感器分子 $\mathrm{S1}$ 对 $\mathrm{CN}^{-}$识别的抗干扰图

Figure 6 Fluorescence intensity changes of the $\mathbf{S 1}$ to $\mathrm{CN}^{-}$in the presence of various test anion in DMSO solution.

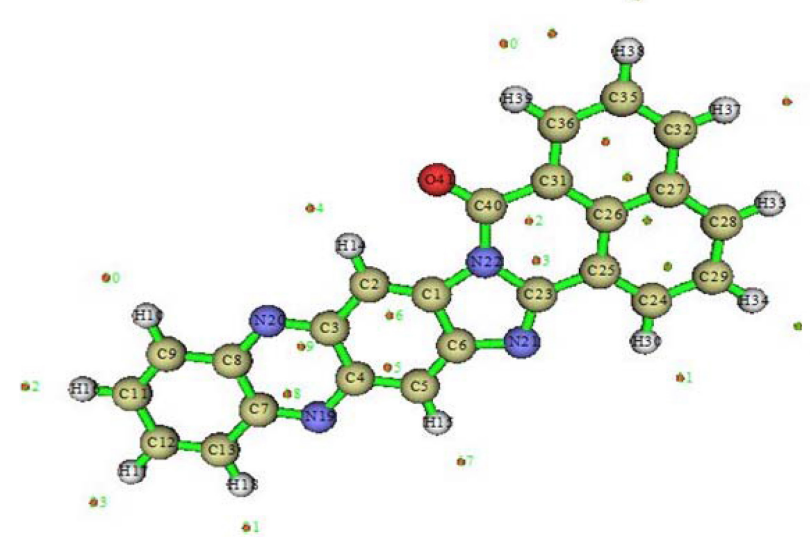

图 7 苂光传感器 $\mathbf{S 1}$ 分子表面静电势分析图

Figure 7 Molecular surface electrostatic potential analysis diagram of fluorescent sensors S1

为了进一步证明氰根加成产物为加成水解产物, 分 别对荧光传感器分子 $\mathbf{S 1}, \mathbf{S 1}+\mathrm{NaCN}$ 和 $\mathbf{S 1}+\mathrm{NaOH}$ 进行 了紫外对比实验(图 8). 从紫外对比图中可以看到, $\mathbf{S 1 +}$ $\mathrm{NaCN}$ 和 $\mathbf{S 1}+\mathrm{NaOH}$ 的紫外吸收峰相似, 说明 $\mathbf{S 1}+$ $\mathrm{NaCN}$ 和 $\mathbf{S 1}+\mathrm{NaOH}$ 体系中有相同的物质产生, $\mathbf{S 1}$ 的碱 性水解产物为开环的羧酸钠盐.

根据以上各实验, 我们认为荧光传感器分子 S1 识 别 $\mathrm{CN}^{-}$机理, 如 Scheme 2 所示: 首先由 $\mathrm{CN}^{-}$对传感器分 子 $\mathrm{S} 1$ 羰基位亲核加成, $\mathrm{C}-\mathrm{N}$ 键断裂生成酰基氭，后者 不稳定发生水解生成羧酸, 最后羧基氢与氮上的钠离子 发生交换生成相应的钠盐. 


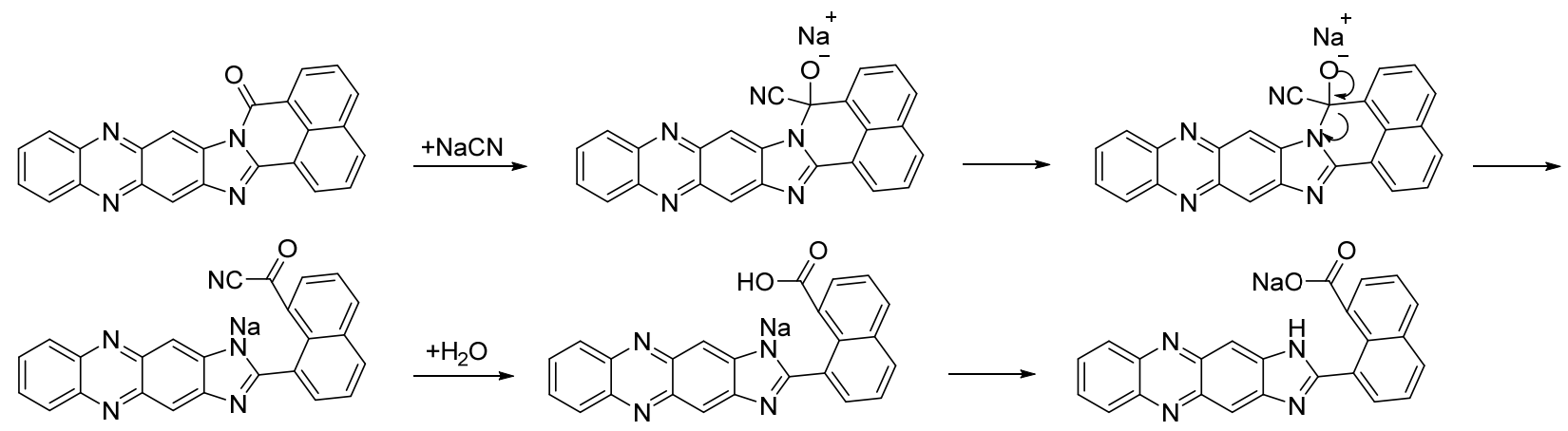

图式 2 苂光传感器分子 $\mathbf{S 1}$ 识别 $\mathrm{CN}^{-}$的机理图

Scheme 2 Proposed mechanism of $\mathbf{S 1}$ for $\mathrm{CN}^{-}$

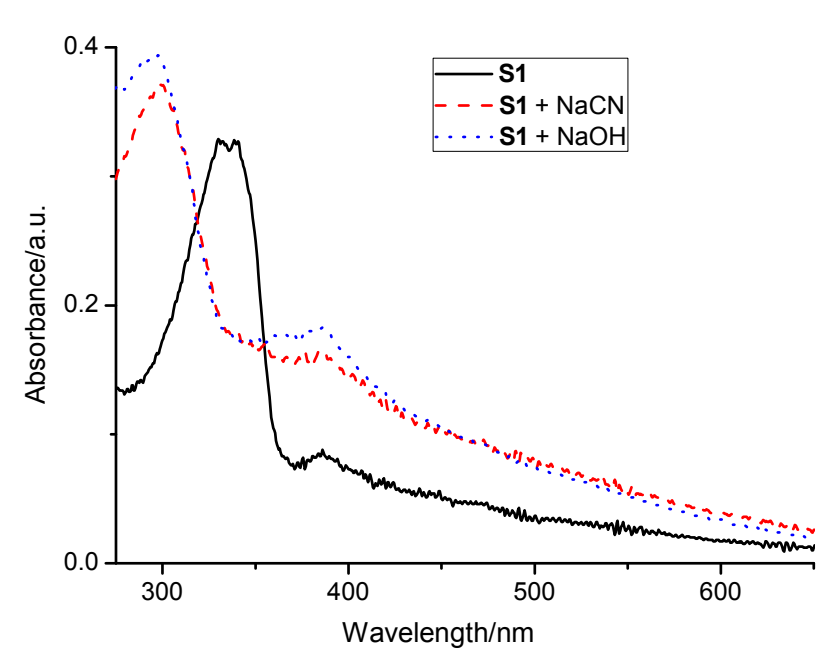

图 8 荧光传感器分子 $\mathrm{S1}, \mathrm{S1}+\mathrm{CN}^{-}$和 $\mathrm{S1}+\mathrm{OH}^{-}$的紫外吸收光 谱对比图

Figure 8 Comparison diagram of chemosensor $\mathbf{S 1}, \mathrm{S} 1+\mathrm{CN}^{-}$ and $\mathbf{S 1}+\mathrm{OH}^{-}$in UV-Absorption

\section{7 荧光传感器分子 $\mathrm{S} 1$ 对 $\mathrm{CN}^{-}$的最低检测限与荧光 量子产率的测定}

在室温条件下, 我们通过向传感器分子 S1 的 DMSO 溶液加入不同浓度的 $\mathrm{CN}^{-}$后, 测定荧光光谱的变 化, 并利用 $3 \mathrm{sB} / \mathrm{S}^{[52]}$ 计算其最低检测限, 得到荧光光谱 的最低检测限为 $9.96 \times 10^{-7} \mathrm{~mol} / \mathrm{L}$. 这一数值低于世界 卫生组织 $(\mathrm{WHO})$ 所规定的饮用水中氧离子的含量 $(<$ $1.9 \times 10^{-6} \mathrm{~mol} / \mathrm{L}$ ). 通过与表 3 中已报道的 $\mathrm{CN}^{-}$探针最低 检测限比较, 发现 $\mathbf{S 1}$ 对 $\mathrm{CN}^{-}$的检测具有检测限较低的 优点, 有一定的实际应用价值. 此外, 我们以硫酸奎宁 做标准参比物, 对 $\mathbf{S 1}$ 与 $\mathrm{CN}^{-}$反应前后的苂光量子产率 做了测定 ${ }^{[53]}$. 其苂光量子产率分别为 0.701 和 0.211 , 说 明 $\mathrm{S} 1$ 与 $\mathrm{CN}^{-}$反应后苂光量子产率明显降低.

\section{8 负载 $\mathrm{S} 1$ 的固体材料制备及其应用}

为了使荧光传感器分子 $\mathbf{S 1}$ 识别 $\mathrm{CN}^{-}$能够在实际中 得到更加简便易行的应用, 我们将 $\mathbf{S 1}$ 固载于硅胶上, 并
表 3 荧光探针识别 $\mathrm{CN}^{-}$比较表

Table 3 Comparison table of current chemosensor with other reported chemosensors for recognition of $\mathrm{CN}^{-}$

\begin{tabular}{cl}
\hline Ref. & $\mathrm{LOD} /\left(\mathrm{mol} \cdot \mathrm{L}^{-1}\right)$ \\
\hline This work & $9.96 \times 10^{-7}$ \\
{$[54]$} & 未给出 \\
{$[3]$} & $1.4 \times 10^{-6}$ \\
{$[55]$} & $4.93 \times 10^{-5}$ \\
{$[56]$} & $1.811 \times 10^{-5}$ \\
{$[57]$} & $1.0 \times 10^{-6}$ \\
\hline
\end{tabular}

用于固体 $\mathrm{NaCN}$ 识别和 $\mathrm{NaCN}$ 水溶液的识别: 称取 $0.0054 \mathrm{~g}$ 荧光传感器分子 $\mathbf{S 1}$, 然后加入到 $0.6 \mathrm{~g}$ 硅胶 (100 200 目, 无色粉末)中, 倒入研针研磨，直到研磨 均匀后倒出三分之一固体粉末, 我们可以观察到此时固 体粉末为淡黄色，在 $365 \mathrm{~nm}$ 三用紫外分析仪下发出亮 黄色苂光. 再从研针中倒出三分之一粉末，加入 0.0024 $\mathrm{g} \mathrm{NaCN}$ 后继续研磨，使其充分被研磨后，倒出粉末，我 们可以观察到粉末颜色略微变化, 相应的在 $365 \mathrm{~nm}$ 三 用紫外分析仪下苂光由黄色变为红色(图 9). 很显然荧 光传感器分子 $\mathbf{S 1}$ 可以识别固体 $\mathrm{NaCN}$. 最后, 向研针中 剩余的三分之一粉末中, 加入 $\mathrm{NaCN}$ 水溶液, 接着在 $365 \mathrm{~nm}$ 三用紫外分析仪下观察苂光颜色，发现相应的 荧光颜色变为红色，说明该固体材料可以直接识别水相 中的氰根离子.

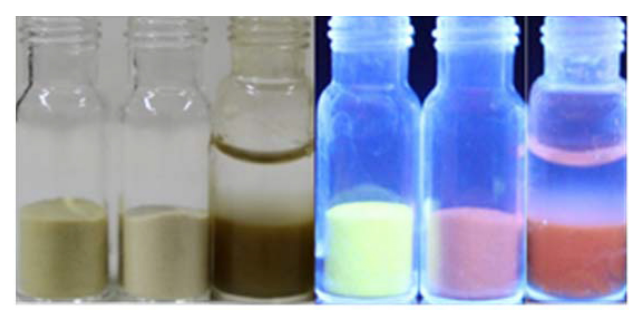

图 9 荧光传感器分子 $\mathbf{S 1}$ 固体材料识别固体 $\mathrm{NaCN}$ 和 $\mathrm{NaCN}$ 水溶液效果图

Figure 9 Fluorescence chemosensor S1 solid material detecting $\mathrm{NaCN}$ in solid and aqueous solution 


\section{2 结论}

本文利用简便方法合成了一种新型荧光传感器分 子 S1, 对其合成条件进行了优化. 然后研究了 S1 在 DMSO 中的苂光光谱性质及其对 $\mathrm{CN}^{-}$的荧光识别响应 性. 研究结果表明: 当加入不同种类的阴离子后, 只有 $\mathrm{CN}^{-}$离子使溶液的荧光颜色由黄色变为橘红色, 表现出 专一选择性识别效果. 同时, 抗干扰实验结果显示, S1 具有良好的抗干扰性能. 在这些实验结果的基础上, 我 们探讨了 $\mathrm{S} 1$ 识别 $\mathrm{CN}^{-}$的机理, 以及传感器分子 $\mathrm{S} 1$ 对 $\mathrm{CN}^{-}$的荧光光谱最低检测限, 发现 $\mathbf{S} 1$ 识别 $\mathrm{CN}^{-}$是一种 新的反应型识别机理, 其最低检测限为 $9.96 \times 10^{-7}$ $\mathrm{mol} / \mathrm{L}$. 最后将其负载于硅胶上, 制备了一种新型固体 识别材料, 该材料不仅可以识别固体 $\mathrm{NaCN}$, 而且可以 识别纯水相中的 $\mathrm{CN}^{-}$根离子, 为氧根离子的识别提供了 行之有效的新方法，具有一定的实际应用价值.

\section{3 实验部分}

\section{1 仪器与试剂}

邻苯二胺、浓盐酸、浓硫酸、硫酸奎宁、 $\mathrm{FeCl}_{3} \cdot 6 \mathrm{H}_{2} \mathrm{O}$ 、 氢氧化钠、 1,8 - 䒬二酸䣶、乙醇、氮氮二甲基甲酰胺 (DMF)、二甲基亚砜(DMSO)均为阿拉丁分析纯级试剂.

${ }^{1} \mathrm{H}$ NMR 使用 Mercury-600BB 型核磁共振仪测定, TMS 为内标; IR 使用 Digilab FTS-3000 FT-IR 型红外光 谱仪 $(\mathrm{KBr}$ 压片) 测定; 熔点使用 X-4 数字显示显微熔点 测定仪测定(温度计未校正); 三用紫外分析仪(上海安亭 电子仪器厂); 紫外光谱使用日本岛津 UV-2550 紫外-可 见吸收光谱仪测定, 测量使用光程为 $1 \mathrm{~cm}$ 的石英比色 皿; 荧光光谱使用 RF-5301 型荧光光谱仪 (日本 Shimadzu 公司); ZAB-HS 型质谱仪(英国 VG 公司).

\section{2 实验方法}

\subsubsection{2,3-二氨基吩嗪的合成}

将 $50 \mathrm{mmol}$ 邻苯二胺 $(5.4 \mathrm{~g}$ )加入 $500 \mathrm{~mL}$ 的圆底烧 瓶中. 然后, 将 $8.33 \mathrm{~mL}$ 浓盐酸稀释于 $250 \mathrm{~mL}$ 蒸馏水中, 将此稀释后的盐酸溶液加入到 $500 \mathrm{~mL}$ 的圆底烧瓶中, 电磁摚拌使邻苯二胺完全溶解. 将 $58 \mathrm{~g} \mathrm{FeCl}_{3} \cdot 6 \mathrm{H}_{2} \mathrm{O}$ 溶 解于 $75 \mathrm{~mL}$ 蒸馏水中, 然后, 用滴液漏斗缓慢滴加至上 述溶液当中(有红色沉淀生成). 滴加完毕后, 室温摚拌 反应 $24 \mathrm{~h}$. 待反应结束后, 抽滤, 并用 $3 \mathrm{~mol} / \mathrm{L}$ 的盐酸溶 液洗涤固体 3 5 次(洗去 $\mathrm{FeCl}_{3}$ 和未反应的邻苯二胺), 将所得的红色固体溶于 $250 \mathrm{ml}$ 沸水中, 再加入 $75 \mathrm{~mL} 2$ $\mathrm{mol} / \mathrm{L}$ 的 $\mathrm{NaOH}$ 溶液, 产生黄色沉淀, 搅拌 $15 \mathrm{~min}$ 左右, 沉降 $1 \mathrm{~h}$, 待溶液冷却至室温, 抽滤得到橘黄色沉淀. 将 所得沉淀用蒸馏水洗涤 2 3 次, 烘干. 得 2,3-二氨基吩 嗪 ${ }^{[58]}$, 产率 $75 \%$. m.p. $>300{ }^{\circ} \mathrm{C}$.
3.2.2 荧光传感器分子 $\mathrm{S} 1$ 的合成及结构表征

将 $0.2216 \mathrm{~g}(1.0 \mathrm{mmol}) 2,3$-二氨基吩嗪和 $0.2453 \mathrm{~g}$ (1.2 mmol) 1,8 -荎二甲酸酐置于 $50 \mathrm{~mL}$ 圆底烧瓶中, 加 入 $20 \mathrm{~mL}$ DMSO 后, 搅拌, 使其完全溶解. 将此混合溶 液于 $130{ }^{\circ} \mathrm{C}$ 油浴锅中, 加热, 回流 $24 \mathrm{~h}$. 待反应结束后, 将反应瓶冷却至室温, 抽虑, 得到褐色沉淀, 用热的乙 醇溶液洗涤沉淀 2 3 次, 烘干, 得到产物 S1, 产率为 63.4\%. m.p. $>300{ }^{\circ} \mathrm{C} ;{ }^{1} \mathrm{H}$ NMR (DMSO- $d_{6}, 600 \mathrm{MHz}$ ) $\delta$ : $8.67 \sim 8.45(\mathrm{~d} 2 \mathrm{H}, \mathrm{ArH}), 8.00 \sim 7.80(\mathrm{~m} \mathrm{4H}, \mathrm{ArH}), 8.67 \sim$ $8.45(\mathrm{~m} 6 \mathrm{H}, \mathrm{ArH}) ;{ }^{13} \mathrm{C}$ NMR (DMSO- $\left.d_{6}, 150 \mathrm{MHz}\right) \delta$ : 162.73, 161.14, 135.82, 132.89, 127.99, 119.48; IR (KBr) $v: 1771,1739,1580,1510,1304,1500 \mathrm{~cm}^{-1}$; HRMS calcd for $\mathrm{C}_{24} \mathrm{H}_{13} \mathrm{~N}_{4} \mathrm{O}[\mathrm{M}+\mathrm{H}]^{+}$372.1011, found 373.1084.

辅助材料(Supporting Information) S1 的核磁氢谱、核 磁碳谱及高分辨质谱. 这些材料可以免费从本刊网站 (http://sioc-journal.cn/)上下载.

\section{References}

[1] Chen, J.-J.; Chen, W.; He, H.; Li, D.-B.; Li, W.-W.; Xiong, L.; Yu, H.-Q. Environ. Sci. Technol. 2013, 47, 1033.

[2] Ahuja, E. G.; Janning, P.; Mentel, M.; Graebsch, A.; Breinbauer, R.; Hiller, W.; Costisella, B.; Thomashow, L. S.; Mavrodi, D. V.; Blankenfeldt, W. J. Am. Chem. Soc. 2008, 130, 17053.

[3] Yang, L.; Li, X.; Qu, Y.; Qu, W.-S.; Zhang, X.; Hang, Y.-D.; Agren, H.; Hua, J.-L. Sen. Actuators, B 2014, 203, 833.

[4] Gu, P.-Y.; Zhao, Y.-B.; He, J.-H.; Zhang, J.; Wang, C.-Y.; Xu, Q.-F.; Lu, J.-M.; Sun, X.-W.; Zhang, Q.-C. J. Org. Chem. 2015, 80, 3030.

[5] Chen, W.; Su, J.-H,; Tian, H. Sci. China: Chem. 2016, 46, 325 (in Chinese).

(陈薇，苏建华，田禾，中国科学：化学, 2016, 46, 325.)

[6] Zhou, H.-T.; Sun, L.; Chen, W.; Tian, G.-J.; Chen, Y.; Li, Y.-R.; Su. J.-H. Tetrahedron 2016, 72, 2300.

[7] Metz, A. E.; Podlesny, E. E.; Carroll, P. J.; Klinghoffer, A. N.; Kozlowski, M. C. J. Am. Chem. Soc. 2014, 136, 10601.

[8] Aggarwal, K.; Khurana, J. M. J. Lumin. 2015, 167, 146.

[9] Wei, T.-B.; Wu, G.-Y.; Shi, B.-B.; Lin,-Q.; Yao, H.; Zhang, Y.-M. Chin. J. Chem. 2014, 32, 1238.

[10] Zhang, P.; Zhang, Y.-M.; Lin, Q.; Yao, H.; Wei, T.-B. Chin. J. Org. Chem. 2014, 34, 1300 (in Chinese). (张鹏, 张有明, 林奇, 姚虹, 魏太保, 有机化学, 2014, 34, 1300.)

[11] Li, C.-W.; Yang, D.; Yin, B.; Guo, Y. Chin. J. Org. Chem. 2016, 36, 787 (in Chinese) (李长伟，杨栋，尹兵，郭媛，有机化学, 2016, 36, 787.)

[12] Gao, G.-B.; Gong, D.-J.; Zhang, M.-X.; Sun, T.-L. Acta. Chim. Sinica. 2016, 74, 363 (in Chinese). (高冠斌, 龚德君, 张明曦, 孙涛垒, 化学学报, 2016, 74, 363.)

[13] Zhang, Y.-P.; You, C.-X.; Yang, Y.-S.; Liu, X.-Y.; Guo, H.-C.; Dong, Y.-Y. Chin. J. Org. Chem. 2016, 36, 1401 (in Chinese). (张应鹏, 尤彩霞, 杨云裳, 刘小育, 郭慧琛, 董玉莹, 有机化学, 2016, 36, 1401.)

[14] Su, N.; Yang, M.-P.; Meng, W.-P.; Yang, B.-Q. Chin. J. Org. Chem. 2015, 35, 175 (in Chinese). (苏娜，杨美盼，孟文斐，杨秉勤，有机化学, 2015, 35, 175.)

[15] Yu, H.-B.; Li, H.-L.; Zhang, X.-F.; Xiao, Y.; Fang, P.-J.; Lü, C.-J.; 
Hou, W. Acta Chim. Sinica 2015, 73, 450 (in Chinese). (于海波, 李红玲, 张新富, 肖义, 方沛菊, 吕春娇, 侯伟, 化学 学报, 2015, 73, 450.)

[16] Zang, L.-B.; Wei, D.-Y.; Wang, S.-C.; Jiang, S.-M. Tetrahedron 2012, 68, 636 .

[17] Gupta, A. S.; Garg, A.; Paul, K.; Luxami, V. J. Lumin. 2016, 173, 165.

[18] Jo, J. Y.; Olasz, A.; Chen, C. H.; Lee, D. J. Am. Chem. Soc. 2013, $135,3620$.

[19] Yan, L.-R.; Yang, M.-P.; Leng, X.; Zhang, M.; Long, Y.; Yang, B.-Q. Tetrahedron 2016, 72, 4361.

[20] Yoo, M.; Park, S.; Kim, H. J. Sen. Actuators, B 2015, 220, 788.

[21] Kim, D.; Na, S.; Y.; Kim, H. J. Sen. Actuators, B 2016, 226, 227.

[22] Wang, S. T.; Chir, J. L.; Jhong, Y.; Wu, A. T. J. Lumin. 2015, 167, 413.

[23] Yang, L.; Li, X.; Yang, J.-B.; Qu, Y.; Hua, J.-L. ACS Appl. Mater. Interfaces 2013, 5, 1317.

[24] Yang, L.; Zhang, X.; Qu, W.-S.; Hua, J.-L. Imaging Sci. Photochem. 2014, 32, 1 (in Chinese). (杨林, 张潇, 矍卫松, 花建丽, 影像科学与光化学, 2014, 32, 1.)

[25] Wang, F.; Wang, L.; Chen, X. Q.; Yoon, J. Y. Chem. Soc. Rev. 2014, $43,12$.

[26] Dagiliene, M.; Martynaitis, V.; Krisiucniene, V.; Krikstolaityte, S.; Sackus, A. ChemistryOpen 2015, 4, 363.

[27] Wang, K.-N.; Ma, L.; Liu, G.-Q.; Cao, D.-X.; Guan, R.-F.; Liu, Z.-Q. Dyes Pigm. 2016, 126, 104.

[28] Lee, K. S.; Kim, H. J.; Kim, G. H.; Shin, I.; Hong, J. I. Org. Lett. 2008, 10, 49.

[29] El-Shishtawy, R. M.; Al-Zahrani, F. A. M.; Al-amshany, Z. M.; Asiri, A. M. Sen. Actuators, B 2017, 240, 288.

[30] Meng, Q.-T. Ph.D. Dissertation, Dalian University of Technology, Dalian, 2011 (in Chinese). (孟庆涛, 博士论文, 大连理工大学, 大连, 2011.)

[31] Shi, B.-B.; Zhang, P.; Wei, T.-B.; Yao, H.; Lin, Q.; Liu, J.; Zhang, Y.-M. Tetrahedron 2013, 69, 7981 .

[32] Shi, B.-B.; Zhang, Y.-M.; Wei, T.-B.; Lin, Q.; Yao, H.; Zhang, P.; You, X.-M. Sens. Actuators, B 2014, 190, 555.

[33] Lin, Q.; Zhu, X.; Chen, P.; Fu, Y. P.; Zhang, Y. M.; Wei, T. B. Acta Chim. Sinica 2013, 71, 1516 (in Chinese).

(林奇, 朱金金, 陈佩, 符永鹏, 张有明, 魏太保, 化学学报, 2013, $71,1516$.

[34] Gao, G.-Y.; Qu, W.-J.; Shi, B.-B.; Lin, Q.; Yao, H.; Yang, W.-L.; Zhang, Y.-M.; Wei, T.-B. Spectrochim. Acta, Part A 2014, 121, 514.

[35] Gao, G.-Y.; Qu, W.-J.; Shi, B.-B.; Lin, Q.; Yao, H.; Zhang, Y.-M.; Chang, J.; Cai, Y.; Wei, T.-B. Sens. Actuators, B 2015, 213, 501.

[36] Hu, J.-Y.; Liu, R.; Zhu, X.-L.; Cai, X.; Zhu, H.-J. Chin. Chem. Lett. $\mathbf{2 0 1 5}, 26,339$
[37] Wei, T.-B.; Li, W.-T.; Li, Q.; Su, J.-X.; Qu, W.-J. Lin, Q.; Yao, H.; Zhang, Y.-M. Tetrahedron Lett. 2016, 57, 2767.

[38] Wei, T.-B.; Li, W.-T.; Li, Q.; Qu, W.-J.; Li, H.; Yan, G.-T.; Lin, Q.; Yao, H.; Zhang, Y.-M. RSC Adv. 2016, 6, 43832.

[39] Li, W.-T.; Wu, G.-Y.; Qu, W.-J.; Li, Q.; Lou, J.-C.; Lin, Q.; Yao, H.; Zhang, Y.-M.; Wei, T.-B. Sens. Actuators, B 2017, 239, 671.

[40] Zhang, Y.-M.; Wang, Y.-L.; Lin, Q.; Wang, D.-D.; Wei, T.-B. Chin. J. Org. Chem. 2009, 29, 575 (in Chinese). (张有明, 王雅琳, 林奇, 王丹丹, 魏太保, 有机化学, 2009，29, 575.)

[41] Wei, T.-B.; Wang, J.; Luo, R.; Zhang, Y.-M. Chin. J. Org. Chem. 2007, 27, 1381 (in Chinese).

(魏太保, 王军, 罗榕, 张有明, 有机化学, 2007, 27, 1381.)

[42] Zhao, J.; Li, J.; Gao, J.; Kjelleberg, S. L. A.; Loo, S. C. J.; Zhang, Q. J. Heterocycl. Chem. 2015, 52, 1699.

[43] Sahoo, P. K.; Giri, C.; Haldar, T. S.; Puttreddy, R.; Rissanen, K.; Mal, P. Eur. J. Org. Chem. 2016, 1283.

[44] Liu, X, X.; Weinert, Z. J.; Sharafi, M.; Liao, C. Y.; Li, J. N.; Schneebeli, S. T. Angew. Chem., Int. Ed. 2015, 54, 12772.

[45] Miao, S. B.; Bangcuyo, C. G.; Smith, M. D.; Bunz, U. H. F. Angew Chem., Int. Ed. 2006, 118, 677.

[46] Wu, J.-S.; Rui, X.-H.; Long, G.-K.; Chen, W.-Q.; Yan, Q.-Y.; Zhang, Q.-C. Angew. Chem., Int. Ed. 2015, 54, 7354.

[47] Hu, J. W.; Zhang, G. H.; Shih, H. H.; Jiang, X. Q.; Suna, P. P.; Cheng, C. H. J. Organomet. Chem. 2008, 693, 2798.

[48] Lucas, M.-D.; Wang, J.-F.; Nick, A.; Li, H.; Liu, T-B.; Pang, Y. J. Phys. Chem. B 2016, 120, 766.

[49] Yang, J.-R.; Fang, H.-P.; Gao, Y. J. Phys. Chem. Lett. 2016, 7, 1788.

[50] Zhou, H.-T.; Sun, L.; Chen, W.; Tian, G.-J.; Chen, Y.; Li, Y.-R.; Su, J.-H. Tetrahedron 2016, 72, 2300.

[51] Zhou, H.-T.; Mei, J.; Chen, Y.-A.; Chen, C.-L.; Chen, W.; Zhang, Z.-Y.; Su, J.-H.; Chou, P.-T.; Tian, H. Small 2016, 12, 6542.

[52] Yang, Y.-T.; Yin, C.-X.; Huo, F.-J.; Chao, J.-B.; Zhang, Y.-B.; Cheng, F.-Q. Sens. Actuators, B 2014, 193, 220.

[53] Li, Q.; Zhang, J.-H.; Cai, Y.; Qu, W.-J.; Gao, G.-Y.; Lin, Q.; Yao, H.; Zhang, Y.-M.; Wei, T.-B. Tetrahedron 2015, 71, 857.

[54] Shive, M.-S.-C.; Tanuja, B.; Bhaskar, G. Tetrahedron Lett. 2008, 49, 6646.

[55] Celso, R. N.; Leandro, G. N.; Vanderlei, G. M. Anal. Chem. 2015, 87,362 .

[56] Ji, H. K.; Seong, Y. L.; Hye, M. A.; Cheal, K. Sens. Actuators, B 2017, 242, 25.

[57] Duan, Y.-L.; Zheng, Y.-S. Talanta 2013, 107, 332.

[58] Shi, B.-B. M.S. Thesis, Northwest Normal University, Lanzhou, 2014 (in Chinese).

(史兵兵, 硕士论文, 西北师范大学, 兰州, 2014.) 